\title{
BMJ Open Factors associated with the responsive behaviours of older adults living in long-term care homes towards staff: a systematic review protocol
}

\author{
Yuting Song, ${ }^{1}$ Matthias Hoben, ${ }^{1}$ Lori Weeks, ${ }^{2}$ Anne-Marie Boström, ${ }^{\oplus 1,3,4}$ \\ Zahra S Goodarzi, ${ }^{5,6,7}$ Janet Squires, ${ }^{8,9}$ Helen Doan, ${ }^{1}$ Adrian Wagg, ${ }^{10}$ \\ R Colin Reid, ${ }^{11}$ Carole Estabrooks ${ }^{1}$
}

To cite: Song Y, Hoben M, Weeks L, et al. Factors associated with the responsive behaviours of older adults living in long-term care homes towards staff: a systematic review protocol. BMJ Open 2019;9:e028416. doi:10.1136/ bmjopen-2018-028416

- Prepublication history and additional material for this paper are available online. To view these files, please visit the journal online (http://dx.doi. org/10.1136/bmjopen-2018028416).

Received 7 December 2018 Revised 25 April 2019 Accepted 29 April 2019

Check for updates

(C) Author(s) (or their employer(s)) 2019. Re-use permitted under CC BY-NC. No commercial re-use. See rights and permissions. Published by BMJ.

For numbered affiliations see end of article.

Correspondence to

Dr Yuting Song;

yuting.song@ualberta.ca

\section{ABSTRACT}

Introduction In the last decade, increasing research interest has been expressed in responsive behaviours of older adults living in long-term care (LTC) homes, including nursing homes and assisted living facilities. Responsive behaviours are not only a sign of underlying unmet needs, but when directed against (towards) paid staff can lead to decreased quality of work life, and may contribute to lower quality of care. In this systematic review, we aim to synthesise empirically based quantitative and qualitative evidence on factors and stakeholder (eg, staff and family members) experiences of factors associated with the responsive behaviours of people living in LTC directed towards staff.

Methods and analysis This study will be a systematic review of published and 'grey' literature. Twelve bibliographical databases will be searched, and for each database, we will use appropriate subject headings and keywords that cover two concepts: LTC and responsive behaviour. No publication date or language filter will be used. The title and abstract of each extracted record will be screened, followed by screening of full text of included papers. Then data extraction and quality assessments will be undertaken. Each stage will be completed independently by pairs of authors. For quantitative studies, meta-analysis will be conducted if pooling is possible; otherwise, a critical narrative analysis will be conducted. For qualitative studies, thematic analysis will be conducted. Factors will then be organised at the individual, interpersonal, institutional and larger societal levels. Sensitivity analysis will be conducted to explore the influence of risk of bias and publication bias on the results. Subgroup analysis will be conducted for people who live with dementia and those who do not.

Ethics and dissemination Ethics approval is not required for this systematic review. The results of this study will be disseminated via peer-reviewed publication and presentation at professional conferences.

\section{INTRODUCTION}

In the last decade increasing research interest has been expressed in responsive behaviours of older adults living in long-term care (LTC)
Strengths and limitations of this study

- This study protocol is informed by the Cochrane Collaboration systematic review methods and adheres to the Preferred Reporting Items for Systematic Reviews and Meta-Analyses Protocols.

- Two reviewers will independently participate throughout the research process including identifying studies for inclusion, data extraction and quality assessment.

- The ecological model will be used to facilitate the organisation and synthesis of results to inform the development of appropriate interventions.

- This review is critical and timely to informing the development and systematic evaluation of effective interventions to prevent and manage responsive behaviours experienced by long-term care staff.

homes, defined here as nursing homes and assisted living facilities. We define responsive behaviours as verbal or physical actions that can be disruptive, distressing or challenging to persons in the environment (eg, other people living in LTC homes, staff and family members). ${ }^{12}$ Researchers have referred to these behaviours as aggressive or challenging behaviours. ${ }^{3}$ However, these terms suggest problems with persons exhibiting the behaviours, thus, do not reflect a person-centred care approach. Instead, we believe that, aligning with the language guideline published by the Alzheimer's Society of Canada, these behaviours are meaningful responses to the environment and reveal underlying concerns, such as pain, loneliness or not wanting personal care. ${ }^{4}$ In this review, therefore, we use the term responsive behaviours, a person-centred term preferred by people living with dementia and their family members. ${ }^{15}$ Of note, because most responsive behaviours occur during 
direct care, ${ }^{46}$ 'resistance to care' is regarded as a type of responsive behaviours in this paper, which aligns with the definition for aggressive behaviour of the Resident Assessment Instrument - Minimum Data Set 2.0. ${ }^{7}$ Despite the increasing research interest in this area, a comprehensive understanding is lacking regarding the multiple factors associated with responsive behaviours towards LTC staff. We address this knowledge gap through this review as a first step to developing and evaluating effective interventions to reduce these behaviours.

\section{Prevalence of responsive behaviours of people living in LTC homes}

Although researchers used different measures, they consistently reported a high prevalence of responsive behaviours in LTC homes, and even higher prevalence among persons living with cognitive impairment who account for the majority of the population in these settings. ${ }^{8}$ A nursing home study using the Resident Assessment Instrument - Minimum Data Set 2.0 revealed that across Canadian provinces, between $26 \%$ and $66 \%$ of individuals exhibited responsive behaviours (ie, verbal abuse, physical abuse, socially inappropriate behaviours, resistance to care) for at least 1 to 3 days in the past 7 days. ${ }^{10}$ A study using a national survey of assisted living facilities in the USA revealed that among the whole population, with or without cognitive impairment, $10 \%$ exhibited verbally abusive behaviour and $8 \%$ exhibited physical aggression, while $23 \%$ of people living with severe cognitive impairment exhibited verbal and physical aggression. ${ }^{11}$ Yet, the rates of responsive behaviours in LTC are likely underreported. For example, staff perceive actual or potential organisational barriers to reporting, such as a perception of inaction on the part of administration or of being stigmatised in the workplace. ${ }^{12}$ We therefore do not have a complete understanding of the full scope of responsive behaviours exhibited by people living in LTC homes.

\section{Impact of responsive behaviours of people living in LTC homes towards staff}

Responsive behaviours can result in harm to the person exhibiting the behaviours, staff or other persons living in LTC. Researchers have studied the significant impact of responsive behaviours, such as injuries and deaths of other people living in LTC homes. ${ }^{13-15}$ Responsive behaviours often result from miscommunication and invasion of space. ${ }^{13}$ Unlike other types of responsive behaviours, most of those exhibited towards staff occur during direct care, especially during morning care when frontline staff provide care for activities of daily living such as toileting, bathing and mouth care. ${ }^{46}$ The high frequency of close interaction during direct care poses uniquely high risks to staff. An analysis of a nationwide care aide survey in the USA revealed that, in the previous year, $34 \%$ of certified nursing assistants reported experiencing physical injuries resulting from responsive behaviours of people living in LTC. ${ }^{3}$ A recent study including a representative sample of
Swiss nursing homes revealed that over a 4-week period, $66 \%, 42 \%$ and $15 \%$ of care workers had experienced verbal, physical and sexual responsive behaviours, respectively. ${ }^{16}$ Experiencing these behaviours can lead to feelings of incompetence or burnout as well as negative feelings of staff towards the individual exhibiting the behaviours. ${ }^{17}$ The presence of responsive behaviours can lead to staff experiencing a wide variety of harms, including burnout, physical injury, decreased job satisfaction and high job turnover. ${ }^{612}$ Therefore, strategies are needed to enable staff to prevent and manage responsive behaviours while providing care.

\section{Management of responsive behaviours in LTC}

The overuse of medications to manage responsive behaviours and the resulting negative impact on the health and quality of life of people living in LTC homes have been widely acknowledged. ${ }^{18} 19$ For example, one in three individuals in LTC homes in Canada is prescribed antipsychotic drugs $^{20}$ to manage behavioural disturbances and agitation associated with dementia, ${ }^{21}$ and a recent report indicated that $27.6 \%$ of antipsychotics used in LTC homes were potentially inappropriately used. ${ }^{22}$ In response, clinical guidelines have recommended first trying non-pharmacological approaches, such as complementary therapies (eg, aromatherapy, music therapy, exercise), care delivery interventions adopting different care models (eg, dementia care planning, person-centred care and function analysis based intervention). ${ }^{23} 24$ However, systematic reviews of these interventions remain inconclusive about their effectiveness compared with usual care at reducing responsive behaviours in LTC. For example, a systematic review and meta-analysis found that care delivery interventions were not effective, yet the strength of evidence of the included studies was low. ${ }^{23}$ Each of another two systematic reviews reached inconsistent conclusions about the effects of complementary therapies to reduce responsive behaviours. ${ }^{25}{ }^{26}$ However, theoretical bases for the development of the included interventions in these three reviews are consistently lacking. Understanding the multiple interrelated factors associated with responsive behaviours is essential not only for the development of the intervention but also to make a rigorous evaluation of proposed interventions.

\section{Rationale for the current systematic review}

In order to develop and evaluate effective interventions to reduce responsive behaviours of people living in LTC homes towards staff, we first need a comprehensive understanding of the multiple factors that are associated with the occurrence of these behaviours. ${ }^{27}$ A preliminary search of PROSPERO, MEDLINE, the Cochrane Database of Systematic Reviews and the Joanna Briggs Institute Database of Systematic Reviews and Implementation Reports was conducted, and no systematic reviews on this topic were currently underway. We did identify three systematic reviews on related topics. Wharton and Ford ${ }^{27}$ focused on the experiences of family caregivers who experienced 
aggression through caring for a family member with dementia in the community, and they recognised that the experiences, training and context are quite different for caregivers in the home versus other settings, such as LTC homes. Welsh and Bader ${ }^{28}$ reviewed situational variables related to aggression in psychiatric inpatient facilities and identified temporal patterns in incident rates of aggression. Zeller and $\mathrm{Hahn}^{29}$ synthesised studies published between 1998 and 2006 and reported on situations in which aggressive behaviours in nursing homes occur. However, this review was limited to a description of the factors and did not synthesise the extent of the effects. A systematic review synthesising associating factors and the extent of association is therefore warranted.

Responsive behaviours of people living in LTC towards staff depend on the complex interplay between staff, individuals and their environment. ${ }^{29}$ This is consistent with the ecological framework developed by Bronfenbrenner, ${ }^{30} 31$ which proposes that human behaviours are affected by multiple factors that are embedded into a nested structure with different levels (eg, individual level, interpersonal level and community level et al). In addition, this model considers the temporal dimension of these factors ${ }^{32}$ such as changes in roommates or exposure to new staff. This framework is useful in conceptualising how such responsive behaviours are interrelated, and influenced by a variety of factors, such as at the individual level (eg, physical and cognitive health status and communication difficulties of individuals living in LTC homes, staff characteristics), the interpersonal level (eg, relationships between individuals and staff), the institutional level (eg, physical space and facility policies) and the larger societal level (eg, LTC regulations, provincial quality improvement programmes). ${ }^{30} 31$

\section{METHODS AND ANALYSIS \\ Aim}

We will systematically review empirical evidence on factors that are associated with responsive behaviours of older adults living in LTC homes towards paid staff. Specific research questions are:

1. Based on quantitative studies, what factors (eg, physical health of individuals living in LTC, staff training level, the physical space of LTC homes) are associated with responsive behaviours?

2. Based on qualitative studies, what are stakeholders' (eg, persons exhibiting behaviours, staff, family members) experiences of factors that are associated with responsive behaviours?

\section{Design}

This protocol is informed by the systematic review methods of the Cochrane Collaboration. It adheres to the Preferred Reporting Items for Systematic Reviews and Meta-Analyses Protocols (PRISMA-P) ${ }^{33}$ We will conduct a single search with two primary analyses for quantitative and qualitative studies respectively that correspond to the above research questions.

\section{Inclusion criteria}

We will consider empirical studies that report findings about factors or stakeholder experiences of factors associated with responsive behaviours of people living in LTC homes towards staff. This will include articles published in peer-reviewed journals, and in 'grey literature' (ie, government reports, union reports, textbooks, non-peer-reviewed articles and theses).

Other inclusion criteria include:

(1) Settings and participants: We will include studies that were conducted in LTC homes, which are referred to with different terms across countries and jurisdictions. ${ }^{3435}$ These homes will meet all the following requirements: provision of residential care for frail people, primarily older adults; 24hours assistance with activities of daily living or instrumental activities of daily living and where length of stay is usually more than 3 months. LTC homes might have a minority population of younger people. While recognising that different stakeholders can experience responsive behaviours exhibited by people living in LTC homes, in this review we focus on paid staff including care aides, nurses and other healthcare professionals.

(2) Types of measures: In this review, responsive behaviours are defined as any form of verbal, physical or sexual behaviours towards paid staff exhibited by people living in LTC homes. ${ }^{3616}$ We will include studies that report on factors at the individual (eg, level of cognitive impairment), interpersonal (eg, relationship between two parties) and environmental levels (eg, staff training, staff mix, staff level, policies, physical environment). Non-English language articles will be translated.

(3) Study designs: This review will include both quantitative and qualitative empirically based studies. For quantitative studies, we will consider both experimental and quasi-experimental study designs including randomised controlled trials, non-randomised controlled trials, before and after studies and interrupted time-series studies. In addition, observational studies including prospective and retrospective cohort studies, case-control studies and analytical cross-sectional studies will also be considered for inclusion. For qualitative studies, we will include designs including, but not limited to, phenomenology, grounded theory, ethnography, action research and critical and feminist research.

\section{Exclusion criteria}

We will exclude studies that are not empirical (eg, editorials, commentaries), as well as those that only focus on the psychometrical testing of instruments. We will exclude studies that focus on LTC homes that admit primarily younger people. We will also exclude quantitative studies that do not include tests of association (eg, only reporting descriptive statistics) and qualitative studies that do not include participants' (eg, staff, residents, family members) insights into factors that relate to responsive behaviours. 


\section{Search strategy}

Twelve bibliographical databases will be searched: Ovid MEDLINE 1946-, Ovid MEDLINE In-Process \& Other Non-Indexed Citations, Ovid Embase 1988-, Ovid PsycINFO 1987-, EBSCOhost CINAHL, EBSCOhost Abstracts in Social Gerontology, Scopus, Web of Science Core Collection (Science Citation Index 1900-, Social Sciences Citation Index 1900-, Conference Proceedings Citation Index-Science 1990-, Conference Proceedings Citation Index-Social Sciences \& Humanities 1990-), Cochrane Database of Systematic Reviews, Cochrane Central Register of Controlled Trials, ProQuest Sociological Abstracts and Proquest Dissertations and Theses Global. Appropriate subject headings and keywords will be used in the search to retrieve articles that cover two concepts: LTC and responsive behaviour. Subject headings and search operators will be modified for each specific database. We will not limit publication dates or language to the searches. We have consulted a scientific librarian for the synonyms and search strategies for each database and conducted preliminary searches (see online supplementary file 1 for example search strategies from one database). Ancestry searches will be done on the reference lists from all included articles. Also, for study protocols, we will search authors' names to identify results that are published in peer-reviewed journals or 'grey literature'. Articles in any non-English language will be translated.

\section{Screening}

Following the search, all identified citations will be collated and uploaded into Covidence systematic review online software (Veritas Health Information, Melbourne, Australia. Available at http://www.covidence.org) to be used to support the screening process. After duplicates are removed, we will pilot the study screening process to refine and clarify the inclusion criteria and to ensure that the criteria are applied consistently by reviewers. We will randomly select 50 papers from the retrieved searches and each reviewer of the screening team will screen the 50 papers using the inclusion and exclusion criteria specified above. Level of agreement among reviewers will be assessed for each pair of reviewers by calculating Kappa. ${ }^{36}$ In cases where the agreement of reviewers is not $100 \%$, all reviewers will discuss and clarify the criteria until $100 \%$ of agreement is reached, and the inclusion criteria will be refined as needed.

After the piloting process is complete, titles and abstracts of remaining papers will be independently screened by two reviewers against the inclusion criteria for the review. Studies included after the title and abstract screening process will be retrieved in full and their details imported into Covidence. The full text of studies will be independently assessed against the inclusion criteria by two reviewers. Studies that do not meet the inclusion criteria will be excluded and reasons for exclusion will be provided in an appendix. Any disagreements that arise between reviewers will be resolved through discussion; should consensus not be reached, agreements will be sought be gaining the opinion of a third reviewer. The results of the screening process will be reported in full and presented in a PRISMA flow diagram. ${ }^{33}$

\section{Data extraction}

We will use an Excel spreadsheet data extraction form (ie, online supplementary file 2) to guide our data extraction. We will test the data extraction process by having each team member extracting data from the same five included studies, including at least one qualitative study. The extracted data will then be compared and any discrepancies will be discussed as a team prior to moving on to extract data from the remainder of the studies. Two reviewers will independently extract data from each study. Any arising disagreements will be resolved through discussion, or with a third reviewer. Authors of included studies will be contacted to request missing or additional data, where required.

The categories of data extracted will include specific details on:

- Study author(s).

- Year of publication.

- Study title.

- Journal (or type of reference if not a journal paper).

- Country of origin (ie, the country in which included LTC homes are located).

- Research question(s) or objective(s).

- Study design.

- Study setting and sample.

- Responsive behaviour(s) measured and tools/ methods used to measure responsive behaviour (dependent variable(s)).

- Factor(s) assessed for association with responsive behaviours (eg, characteristics of people living in LTC homes, care staff, care units, facilities) and tools/ methods used to assess these factors (independent variable(s)).

- Statistical analyses.

- Main study findings.

Quantitative study findings of interest to this review include:

- Tests of difference, such as; (a) t-tests for independent samples to assess whether the prevalence of responsive behaviours is different in two independent groups of people living in LTC homes (eg, intervention recipients vs control group participants or people with different levels of cognitive impairment), (b) t-tests for dependent samples to assess whether the prevalence of responsive behaviours changes in a group of people living in LTC homes over time (eg, after they underwent an intervention), (c) analyses of variance to test differences in responsive behaviours in more than two groups of people living in LTC homes and (d) tests to assess differences in categorical dependent outcomes (eg, responsive behaviours yes/no or low, medium, high) between groups or over time, such as $\mathrm{X}^{2}$ test, Kruskal-Wallis test, Wilcoxon signed rank test. 
- Bivariate correlations assessing whether or not certain factors are correlated with responsive behaviours (eg, Pearson's correlation coefficient, Spearman's rank correlation coefficient, Kendall's tau correlation coefficient).

- Regression coefficients indicating whether or not certain factors are associated with responsive behaviours, based on simple linear regressions, multiple linear regressions, hierarchical mixed models (for continuous behaviour outcomes such as prevalence of responsive behaviours) or non-linear equivalents such as binary logistical regressions, Poisson regressions, generalised mixed models (for categorical behavioural outcomes).

- Relative risks or ORs to assess whether or not certain factors increase the risks/odds for exhibiting responsive behaviours.

For qualitative studies, we will include themes, categories, patterns, theories and concepts as reported in the studies along with associated illustrations. Specifically, these will include:

- Experiences of staff to whom responsive behaviours were directed, such as their feelings of what happened to them, impact on their work life, impact on their perceptions towards people living in LTC homes and the subsequent care they provide.

- Experiences of other stakeholders' (eg, persons exhibiting behaviours, family members, managers, other individuals living in LTC homes) with responsive behaviours towards staff, such as their feelings about what happened to staff, their perspectives of impact on staff work life and the care that staff provide.

- Explanations of what led to the responsive behaviours towards staff (eg, factors related to the individuals such as pain, discomfort and unmet care needs, staff factors such as stress or feeling overwhelmed, work environment, facility policies).

\section{Assessment of risk of bias}

Each study included in the review will be critically appraised by pairs of reviewers, using the assessment tool appropriate for the design of each included study. Any disagreements that arise between the reviewers will be resolved through discussion, or with a third reviewer.

For randomised clinical trials, non-randomised experiments, cohort studies and case-control studies, we will use the Quality Assessment Tool for Quantitative Studies, ${ }^{37}$ which has established validity and reliability. ${ }^{38}{ }^{39}$ This tool assesses eight domains: selection bias, study design, confounders, blinding, data collection methods, withdrawals and dropouts, intervention integrity and analysis. An overall rating of strong, moderate or weak is assigned based on scores of each domain.

For cross-sectional studies, we will use the rigorously developed AXIS critical appraisal tool. ${ }^{40}$ This tool contains 20 guiding questions relating to the quality of reporting, study design quality and possible introduction of biases.
The reviewer will assign to each guiding question one of three options: yes, no, do not know.

For qualitative studies, the Joanna Briggs Institute standardised critical appraisal instrument for qualitative research will be used. ${ }^{41}$ We will assess the rigour of each included study using 10 guiding questions in areas such as the congruity between the stated philosophical perspective and the research methodology, the research methodology and the research question and the research methodology and data collection methods. Each study will be assigned yes, no or unclear for each guiding question.

All studies, regardless of their methodological quality, will undergo data extraction. However, the judgement based on risk of bias assessment for studies will be reported and results will be discussed in the context of the quality assessment.

\section{Data analysis and synthesis}

If possible, we will statistically pool results of quantitative studies, using random-effects meta-analysis. We will conduct these analyses separately for longitudinal and cross-sectional studies. Statistical pooling is possible if three or more longitudinal studies or three or more cross-sectional studies (a) report the same influencing factor on responsive behaviour, (b) measure this factor in a comparable way (eg, all studies used a comparable measurement tool and report the outcome in the same way), (c) report the same type of responsive behaviour, (d) measure responsive behaviour in a comparable way and (e) report the same type of statistical outcome. Pooling a minimum of two studies can be conducted statistically. ${ }^{42}$ However, a minimum of three studies are required to allow for estimating measures of heterogeneity in addition to estimating the pooled effect for random-effects meta-analysis. ${ }^{43}$ Where possible, we will contact authors of included studies to obtain missing information. We will use STATA V.15 (StataCorp LLC, College Station, Texas) to run random-effects models, which are more appropriate than fixed-effects models if we identify heterogeneity and small numbers of included studies. ${ }^{44}$ We will report pooled effect sizes and their $95 \%$ CIs and we will calculate the $\mathrm{I}^{246}{ }^{47}$ and $\mathrm{H}^{48}$ statistics (including their 95\% CIs) to assess statistical heterogeneity. To assess for publication bias, we will create funnel plots and conduct visual inspections of these funnel plots as well as random regression analyses to test for funnel plot asymmetry. ${ }^{49}$ If necessary, we will adjust our pooled effect sizes for publication bias, using the Duval and Tweedie's trim-and-fill procedures. $^{50}$

Where statistical pooling is not possible, findings will be presented in a narrative form including tables and figures to aid in data presentation. To determine the association of factors with responsive behaviours towards staff, we will apply the following principles to guide our synthesis. ${ }^{51-53}$

1. In total, if fewer than four articles examined the association between one factor and responsive behaviours of people living in LTC homes towards staff, the association is inconclusive. 
2. If four or more articles examined the association between one factor and responsive behaviours of people living in LTC homes towards staff, (1) the association is significant if $60 \%$ or more articles reveal a significant relationship in the same direction, (2) the association is non significant if $60 \%$ or more articles reveal an insignificant relationship or (3) the association is equivocal if fewer than $60 \%$ revealed a significant/insignificant relationship.

3. When a study reports both bivariate and multivariate statistical analyses, we will use multivariate statistics in making the above decisions. Our focus in this review is the association of responsive behaviours with factors at multiple levels; this includes both whether or not an association exists and the strength of the association. Compared with bivariate analyses, multivariate analyses are more rigorous by accounting for confounding variables that can influence the relationship, which supports the selection of multivariate analyses over bivariate analyses should both be reported in the same paper.

For qualitative data, we will use thematic analysis ${ }^{54}$ to synthesise the findings to generate a set of statements that represent that aggregation. This will be followed with categorising the findings by the similarity in meaning across the levels of the ecological model. We will then use these categories to produce a single comprehensive set of synthesised findings that can be used as a basis for evidence-based practice. Where textual pooling is not possible, findings will be presented in narrative form.

Subgroup analysis: Persons with dementia experience unique challenges due to communication difficulties ${ }^{13}$ and have a higher prevalence of responsive behaviours than those without dementia in LTC. ${ }^{11}$ Therefore, subgroup analysis will be conducted with the group of people with dementia and others, using quantitative and qualitative data separately.

The quantitative and qualitative outcomes will be included in a summary of findings table by levels of the ecological model at the individual level, the interpersonal level, the institutional or environmental level ${ }^{3031}$ and the influences of the passage of time. ${ }^{32}$ A summary of quantitative findings table will be created using GRADEpro GDT software. ${ }^{55}$ The GRADE approach for grading the quality of quantitative evidence will be followed. The following information will be presented where appropriate: estimates of relative risk, and a ranking of the quality of the evidence based on study limitations (risk of bias), indirectness, inconsistency, imprecision and publication bias.

The final qualitative synthesised findings will be graded according to the ConQual approach for establishing confidence in the output of qualitative research synthesis and presented in a summary of findings table. ${ }^{57}$ The summary of findings table will include the major elements of the review and will detail development of the ConQual score. Included in the table will be the title, population, phenomena of interest and context for the specific review. Each synthesised finding from the review will then be presented along with the type of research informing it, a score for dependability, credibility and the overall ConQual score.

\section{Patient and public involvement}

Patients and members of the public were not involved in the development of this review protocol.

\section{Protocol amendments}

The protocol is registered at the International Prospective Register of Systematic Reviews (PROSPERO); the registration number is: CRD42019089368. Any amendments to the protocol will be documented on PROSPERO and in the final manuscript.

\section{Strengths and limitations of this study}

The results of this study will contribute to developing effective strategies to reduce these behaviours in LTC settings. Ultimately, our goal is to contribute to the prevention of responsive behaviours of LTC residents, and in particular responsive behaviours towards staff. While there is increased interest in this topic, a preliminary search of the literature indicates that there is limited research available focused on this topic within $\mathrm{LTC}^{27}$ Our inclusion of many study designs will ensure that we will provide the best evidence possible about the factors that contribute to responsive behaviours of residents in LTC.

As rates of responsive behaviours in the LTC setting are likely underreported, we are aware that our results will not reflect the full extent of responsive behaviours that occur in LTC towards staff. In this systematic review, we will carefully report how data were collected from staff and identify potential ways to improve the data collection process to encourage staff to feel comfortable reporting their experiences.

\section{ETHICS AND DISSEMINATION}

Ethics approval is not required for this systematic review. The results of this study will be disseminated via peer-reviewed publication and presentation at professional conferences. As this team is part of the Translating Research in Elder Care (TREC) research team (https:// trecresearch.ca/), results will also be disseminated via the TREC website, newsletter and other media of TREC. The results of this study will contribute to developing effective strategies to reduce these behaviours in LTC settings. By using the ecological model, we will contribute to knowledge about various ways that interventions can be implemented, such as interventions focused at the individual level, the interpersonal level or the environmental level. Ultimately, our goal is to contribute to the prevention of responsive behaviours of people living in LTC homes, and in particular responsive behaviours towards staff. These results may contribute to improved quality of life of people living in LTC homes and improved quality of work life of staff. 
Author affiliations

${ }^{1}$ Faculty of Nursing, University of Alberta, Edmonton, Alberta, Canada

${ }^{2}$ School of Nursing, Dalhousie University, Halifax, Nova Scotia, Canada

${ }^{3}$ Division of Nursing, Karolinska Institutet, Stockholm, Sweden

${ }^{4}$ Theme Aging, Karolinska University Hospital, Huddinge, Sweden

${ }^{5}$ Department of Medicine, University of Calgary, Calgary, Alberta, Canada

${ }^{6}$ Hotchkiss Brain Institute, University of Calgary, Calgary, Alberta, Canada

${ }^{7} 0$ 'Brien Institute for Public Health, University of Calgary, Calgary, Alberta, Canada

${ }^{8}$ School of Nursing, University of Ottawa, Ottawa, Ontario, Canada

${ }^{9}$ Clinical Epidemiology Program, the Ottawa Hospital Research Institute, Ottawa,

Ontario, Canada

${ }^{10}$ Department of Medicine, University of Alberta, Edmonton, Alberta, Canada

${ }^{11}$ Faculty of Health and Social Development, School of Health and Exercise

Sciences, University of British Columbia Okanagan, Kelowna, British Columbia,

Canada

Acknowledgements The authors acknowledge the Translating Research in Elder Care (TREC) 2.0 team for its contributions to this study. Funding was provided by the Canadian Institutes of Health Research (CIHR) and partners in the Ministries of Health in British Columbia, Alberta and Manitoba, as well as, regional health authorities in participating British Columbia and Alberta regions.

Contributors YS, LW and A-MB developed the research questions, the systematic review design, developed the study protocol and are leading this project. YS, LW, A-MB and HD conducted the preliminary search and pilot-tested the search strategies. YS wrote the first draft of the manuscript; MH and LW assisted with drafting Data Extraction and Data Analysis sections. All authors critically read, provided feedback on the manuscript and have approved its submission.

Funding YS conducted this research as part of her post-doctoral work, funded by CE Canada Research Chair (Tier I), Faculty of Nursing, University of Alberta.

Competing interests None declared.

Patient consent for publication Not required.

Provenance and peer review Not commissioned; externally peer reviewed.

Open access This is an open access article distributed in accordance with the Creative Commons Attribution Non Commercial (CC BY-NC 4.0) license, which permits others to distribute, remix, adapt, build upon this work non-commercially, and license their derivative works on different terms, provided the original work is properly cited, appropriate credit is given, any changes made indicated, and the use is non-commercial. See: http://creativecommons.org/licenses/by-nc/4.0/.

\section{REFERENCES}

1. Alzheimer Society of Canada Responsive behaviours. 2017. http:// alzheimer.ca/en/bc/Living-with-dementia/Caring-for-someone/ Understanding-symptoms/Responsive-behaviours

2. Hoben M, Kent A, Kobagi N, et al. Effective strategies to motivate nursing home residents in oral healthcare and to prevent or reduce responsive behaviours to oral healthcare: a systematic review protocol. BMJ Open 2016;6:e011159.

3. Ryden MB. Aggressive behavior in persons with dementia who live in the community. Alzheimer Dis Assoc Disord 1988;2:342-55.

4. Morgan DG, Cammer A, Stewart NJ, et al. Nursing aide reports of combative behavior by residents with dementia: results from a detailed prospective incident diary. J Am Med Dir Assoc 2012:13:220-7.

5. Alzheimer Society of Canada. Person Centered Language Guidelines. 2017. http://alzheimer.ca/sites/default/files/2017-11/Person Centred Language Guidelines-e.pdf

6. Lachs MS, Rosen T, Teresi JA, et al. Verbal and physical aggression directed at nursing home staff by residents. J Gen Intern Med 2013;28:660-7.

7. Perlman CM, Hirdes JP. The aggressive behavior scale: a new scale to measure aggression based on the minimum data set. J Am Geriatr Soc 2008;56:2298-303.

8. Bartfay E, Bartfay WJ, Gorey KM. Prevalence and correlates of potentially undetected dementia among residents of institutional care facilities in Ontario, Canada, 2009-2011. Int J Geriatr Psychiatry 2013;28:1086-94.

9. Livingston G, Barber J, Marston L, et al. Prevalence of and associations with agitation in residents with dementia living in care homes: MARQUE cross-sectional study. BJPsych Open 2017;3:171-8.
10. Hirdes JP, Mitchell L, Maxwell CJ, et al. Beyond the 'iron lungs of gerontology': using evidence to shape the future of nursing homes in Canada. Can J Aging 2011;30:371-90.

11. Zimmerman S, Sloane PD, Reed D. Dementia prevalence and care in assisted living. Health Aff 2014;33:658-66.

12. Edward KL, Ousey K, Warelow $P$, et al. Nursing and aggression in the workplace: a systematic review. Br J Nurs 2014;23:653-9.

13. Caspi E. Aggressive behaviors between residents with dementia in an assisted living residence. Dementia 2015;14:528-46.

14. Shinoda-Tagawa T, Leonard R, Pontikas J, et al. Resident-to-resident violent incidents in nursing homes. JAMA 2004;291:591-8.

15. Caspi E. Deaths as a Result of Resident-to-Resident Altercations in Dementia in Long-Term Care Homes: A Need for Research, Policy, and Prevention. J Am Med Dir Assoc 2016;17:7-11.

16. Stutte K, Hahn S, Fierz K, et al. Factors associated with aggressive behavior between residents and staff in nursing homes. Geriatr Nurs 2017;38:398-405.

17. Holst A, Skär L. Formal caregivers' experiences of aggressive behaviour in older people living with dementia in nursing homes: A systematic review. Int J Older People Nurs 2017:12:e12158.

18. Ballard C, O'Brien J, James I, et al. Quality of life for people with dementia living in residential and nursing home care: the impact of performance on activities of daily living, behavioral and psychological symptoms, language skills, and psychotropic drugs. Int Psychogeriatr 2001;13:93-106.

19. Juola AL, Pylkkanen S, Kautiainen $\mathrm{H}$, et al. Burden of potentially harmful medications and the association with quality of life and mortality among institutionalized older people. J Am Med Dir Assoc 2016;17:276.e9-276.e14.

20. Canadian Institute for Health Information. Antipsychotic use in seniors: An analysis focusing on drug claims. 2009. https://secure. cihi.ca/free_products/antipsychotics_aib_en.pdf

21. Hagen B, Esther CA, Ikuta R, et al. Antipsychotic drug use in Canadian long-term care facilities: prevalence, and patterns following resident relocation. Int Psychogeriatr 2005;17:179-93.

22. Canadian Medical Association. The state of seniors health care in Canada. 2016. https://www.cma.ca/En/Lists/Medias/the-state-ofseniors-health-care-in-canada-september-2016.pdf

23. Jutkowitz E, Brasure M, Fuchs E, et al. Care-delivery interventions to manage agitation and aggression in dementia nursing home and assisted living residents: a systematic review and meta-analysis. $J$ Am Geriatr Soc 2016;64:477-88.

24. Ballard C, Orrell M, YongZhong S, et al. Impact of antipsychotic review and nonpharmacological intervention on antipsychotic use, neuropsychiatric symptoms, and mortality in people with dementia living in nursing homes: a factorial cluster-randomized controlled trial by the Well-Being and Health for People With Dementia (WHELD) Program. Am J Psychiatry 2016;173:252-62.

25. Watson K, Chang E, Johnson A. The efficacy of complementary therapies for agitation among older people in residential care facilities: a systematic review. JBI Libr Syst Rev 2012;10:3414-86.

26. van der Steen JT, Smaling HJ, van der Wouden JC, et al. Musicbased therapeutic interventions for people with dementia. Cochrane Database Syst Rev 2018;7:CD003477.

27. Wharton TC, Ford BK. What is known about dementia care recipient violence and aggression against caregivers? J Gerontol Soc Work 2014:57:460-77.

28. Welsh E, Bader S, Evans SE. Situational variables related to aggression in institutional settings. Aggress Violent Behav 2013;18:792-6.

29. Zeller A, Hahn S, Needham I, et al. Aggressive behavior of nursing home residents toward caregivers: a systematic literature review. Geriatr Nurs 2009;30:174-87.

30. Bronfenbrenner U. Toward an experimental ecology of human development. Am Psychol 1977;32:513-31.

31. Bronfenbrenner U. Ecology of the family as a context for human development: Research perspectives. Dev Psychol 1986;22:723-42.

32. Schaie KW, Schooler C. Social structure and aging: Psychological processes. Hillsdale, NJ: Psychology Press, 1989.

33. Shamseer L, Moher D, Clarke M, et al. Preferred reporting items for systematic review and meta-analysis protocols (PRISMA-P) 2015: elaboration and explanation. BMJ 2015;349:g7647.

34. Kissam S, Gifford DR, Mor V, et al. Admission and continued-stay criteria for assisted living facilities. J Am Geriatr Soc 2003;51:1651-4

35. Sanford AM, Orrell M, Tolson D, et al. An international definition for "nursing home". J Am Med Dir Assoc 2015;16:181-4.

36. Tacconelli E. Systematic reviews: CRD's guidance for undertaking reviews in health care. Lancet Infect Dis 2010;10:226.

37. National Collaborating Centre for Methods and Tools. Quality assessment tool for quantitative studies. Hamilton, ON: McMaster 
University, 2008. Available: http://www.nccmt.ca/resources/search/ 14 [updated 03 Oct 2017].

38. Armijo-Olivo S, Stiles CR, Hagen NA, et al. Assessment of study quality for systematic reviews: a comparison of the Cochrane Collaboration Risk of Bias Tool and the Effective Public Health Practice Project Quality Assessment Tool: methodological research. J Eval Clin Pract 2012;18:12-18.

39. Thomas BH, Ciliska D, Dobbins $\mathrm{M}$, et al. A process for systematically reviewing the literature: providing the research evidence for public health nursing interventions. Worldviews Evid Based Nurs 2004;1:176-84.

40. Downes MJ, Brennan ML, Williams HC, et al. Development of a critical appraisal tool to assess the quality of cross-sectional studies (AXIS). BMJ Open 2016;6:e011458.

41. Institute JB. Joanna Briggs Institute reviewers' manual: 2014 edition. Australia: The Joanna Briggs Institute, 2014.

42. Valentine JC, Pigott TD, Rothstein HR. How many studies do you need? A primer on statistical power for meta-analysis. J Educ Behav Stat 2010;35:215-47.

43. Borenstein M, Hedges L, Higgins J, et al. When does it make sense to perform a meta-analysis. Introduction to meta-analysis 2009:357-64.

44. Brockwell SE, Gordon IR. A comparison of statistical methods for meta-analysis. Stat Med 2001;20:825-40.

45. Kontopantelis E, Reeves D. Performance of statistical methods for meta-analysis when true study effects are non-normally distributed: a comparison between DerSimonian-Laird and restricted maximum likelihood. Stat Methods Med Res 2012;21:657-9.

46. Higgins JP, Thompson SG. Quantifying heterogeneity in a metaanalysis. Stat Med 2002;21:1539-58.
47. Higgins JP, Thompson SG, Deeks JJ, et al. Measuring inconsistency in meta-analyses. BMJ 2003;327:557-60.

48. Mittlböck M, Heinzl H. A simulation study comparing properties of heterogeneity measures in meta-analyses. Stat Med 2006;25:4321-33.

49. Egger M, Davey Smith G, Schneider M, et al. Bias in meta-analysis detected by a simple, graphical test. BMJ 1997;315:629-34.

50. Duval S, Tweedie R. Trim and fill: A simple funnel-plot-based method of testing and adjusting for publication bias in meta-analysis. Biometrics 2000;56:455-63.

51. Squires JE, Hoben M, Linklater S, et al. Job Satisfaction among Care Aides in Residential Long-Term Care: A Systematic Review of Contributing Factors, Both Individual and Organizational. Nurs Res Pract 2015;2015:1-24.

52. Squires JE, Estabrooks CA, Gustavsson P, et al. Individual determinants of research utilization by nurses: a systematic review update. Implement Sci 2011;6:1.

53. Dilig-Ruiz A, MacDonald I, Demery Varin M, et al. Job satisfaction among critical care nurses: A systematic review. Int J Nurs Stud 2018;88:123-34.

54. Pearson A, Rittenmeyer L, Robertson-Malt S. Synthesizing qualitative evidence. Lippincott Williams \& Wilkins Philadelphia, PA, 2011.

55. Guyatt $\mathrm{GH}$, Oxman AD, Vist GE, et al. GRADE: an emerging consensus on rating quality of evidence and strength of recommendations. BMJ 2008;336:924-6.

56. Guyatt $\mathrm{GH}$, Oxman AD, Vist G, et al. GRADE guidelines: 4. Rating the quality of evidence-study limitations (risk of bias). J Clin Epidemiol 2011;64:407-15

57. Munn Z, Porritt K, Lockwood C, et al. Establishing confidence in the output of qualitative research synthesis: the ConQual approach. BMC Med Res Methodol 2014;14:108. 